\title{
A dynamic in-vitro model of the intestinal epithelium for the investigation of P-glycoprotein activity
}

Joana Costa ( $\square$ joana.costa@ing.unipi.it )

Universita degli Studi di Pisa https://orcid.org/0000-0001-9483-1697

Vanessa Almonti

Universita degli Studi di Genova

\section{Ludovica Cacopardo}

Universita degli Studi di Pisa

\section{Daniele Poli}

Universita degli Studi di Pisa

Simona Rapposelli

Universita degli Studi di Pisa

Arti Ahluwalia

Universita degli Studi di Pisa

\section{Research}

Keywords: Curcumin, Caco-2 cells, fluidic systems, P-gp modulation, bioreactors, intestinal in-vitro models

Posted Date: July 7th, 2020

DOl: https://doi.org/10.21203/rs.3.rs-24178/v2

License: (c) (i) This work is licensed under a Creative Commons Attribution 4.0 International License.

Read Full License 


\section{Abstract}

Multidrug resistance is still an obstacle for chemotherapeutic treatments. One of the proteins involved in this phenomenon is the P-glycoprotein, P-gp, which is known to be responsible for the efflux of therapeutic substances from the cell cytoplasm. To date, the identification of a drug that can efficiently inhibit P-gp activity remains a challenge, nevertheless some studies have identified natural compounds suitable for that purpose. Amongst them, curcumin has shown an inhibitory effect on the protein in in vitro studies using Caco-2 cells.

To understand if physiological flow can modulate membrane protein activity, we studied the uptake of a P-gp substrate under static and dynamic conditions. Caco-2 cells were cultured in bioreactors and in Transwells and the basolateral transport of Rhodamine-123 assessed in the two systems as a function of P-gp activity. Experiments were performed with and without pre-treatment of the cells with an extract of curcumin or an arylmethyloxy-phenyl derivative to evaluate the inhibitory effect of the natural substance with respect to a synthetic compound.

The results indicated that the P-gp activity of the cells cultured in the bioreactors was intrinsically lower, and that the effect of both natural and synthetic inhibitors was up modulated by the presence of flow. Our study underlies the fact that the use of more sophisticated and physiologically relevant in vitro models can bring new insights on the therapeutic effects of natural substances such as curcumin.

\section{Background}

Chemotherapy remains the preferred treatment in the case of advanced and/or metastatic neoplasms. Unfavourably, cancer cells have shown the ability to develop resistance to many different antineoplastic drugs, displaying an adaptation commonly mentioned as "pleiotropic resistance" or "multidrug resistance" (MDR) (1). Cellular mechanisms linked to drug-resistance can occur simultaneously or sequentially to pharmacological resistance in cells exhibiting a drug-resistant-phenotype, which can be manifested through the activation of numerous proteins (2). One of the proteins involved in MDR is the membrane glycoprotein (P-gp), which belongs to the class of plasma membrane efflux proteins. P-gp acts like a pump, extracting neutral or weakly basic amphipathic substances, such as chemotherapeutic agents, from cell cytoplasm. In fact, P-gp has been the main pharmacological target in combating MDR for several years (3).

In the human gastrointestinal tract, P-gp is found in high concentrations on the apical surfaces of superficial columnar epithelial cells of the colon and distal small bowel. Its expression appears to increase when the tissues pass into the tumor state, as occurs in the colorectal epithelium (4). Many attempts have been made to identify effective P-gp inhibitors, known as chemosensitizers or MDR modulators, which sensitize resistant cells to the action of cytotoxic drugs. The approaches used up to now have not returned significant results and have failed in clinical trials (1). The first attempts to modulate P-gp were carried out by testing calcium-channel blockers and antisteroids (first-generation 
drugs), however these compounds interfered with several enzyme systems (5). Later, second- and thirdgeneration drugs were developed, but have failed in clinical trials mainly due to unpredictable pharmacokinetic interactions (6).

Alternatively, researchers have tried to find a valid P-gp inhibitor from substances of natural origin. Among the compounds studied, we can cite those deriving from black pepper [piperine, Piper nigrum] (7), sage [Thanshinones, Salvia miltiorrhiza] (8), licorice [glycyrrhetinic acid, Glycyrrhiza glabra], chilli pepper [capsaicin, Capsicum annuum] green tea [(-)-Epigallocatechin gallate, Camellia sinensis] (9) and turmeric [curcumin, Curcuma Longa] (10).

Curcuma Longa has been one of the most intensively studied plant extracts and its action on the intestinal glycoprotein has been investigated in static in vitro models. Figure 1 illustrates the inhibition of P-gp pumping activity in the presence of curcumin.

In a study using intestinal cell monolayers as a model of the gastro-intestinal tract, the authors found that curcumin down regulated the expression and function of intestinal P-gp (11). Specifically, curcumin at a concentration of $30 \mu \mathrm{M}$ increased Rhodamine-123 (Rh-123) (a substrate of P-gp) accumulation by twofold compared to the negative control and reduced efflux by $30 \%$ in the apical site. Ampasavate et al. investigated P-gp modulating effects of extracts prepared from rhizomes of Curcuma Longa and Curcuma sp. "Khamin-oi". They showed that the extract of Curcuma Longa increased Rh-123 uptake in a dose dependent manner, inhibiting the activity of intestinal P-gp. (10).

Both studies were performed using Caco-2 cells, cultured in a Transwell® system, which differentiate to form a polarized epithelial cell monolayer providing a physical and biochemical barrier to the passage of ions and small molecules $(12,13)$. This cell line is widely used across the pharmaceutical industry as an in vitro model of the human small intestinal mucosa to predict the absorption of orally administered drugs (14).

In order to understand how the presence of flow could affect the modulation of P-gp, we studied the inhibition of intestinal P-gp by culturing Caco-2 cells in bioreactors. The systems we used combine a twocompartment cell culture device with apical and basal media flow and have been described by Giusti (Giusti et al. 2014) and Cacopardo (16). Both studies demonstrate that they are capable of better mimicking physiological barriers as they recapitulate the dynamic environment of the intestine. Here we compared the apical efflux of a P-gp substrate after preconditioning with a Curcumin extract and a synthetic compound (an arylmethyloxy-phenyl derivative or APD (17)) which proved to inhibit P-gp activity in a $\left[{ }^{3} \mathrm{H}\right]$-vinblastine transport inhibition test with $\mathrm{IC}_{50}$ of $0.19 \mathrm{mM}$. The experiments were performed in Transwells (traditional static system) and using bioreactors. To the best of our knowledge this is the first application of such devices to the investigation of P-gp inhibition by curcumin.

\section{Methods}


First, we performed a preliminary cytotoxicity test to determine a safe curcumin dose to be employed in the P-gp modulation study. Then, we set-up the culture of Caco-2 cells in the two systems for 21 days, with regular cell monitoring, including TEER and TEEI (Trans Epithelial Electrical Resistance and Impedance respectively). Finally, the P-gp activity in response to treatment with the two different compounds (Curcumin extract and the synthetic compound APD) was assessed through a transport study using a P-gp substrate: rhodamine-123 (Rh-123). Figure 2 schematically illustrates the experiments performed.

\section{Cell culture and subculturing}

Caco-2 cells from the American Type Culture Collection (ATCC) were grown in tissue culture flasks in Dulbecco's Modified Eagle's medium (high glucose) supplemented with 10\% (v/v) heat-inactivated Fetal Bovine Serum (FBS), 1\% (v/v) L-glutamine, 1\% (v/v) non-essential amino acids and 1\% (v/v) penicillin/streptomycin. All reagents were purchased from Sigma-Aldrich, Italy, unless stated otherwise.

The LB2 bioreactors, purchased from IVTech srl, are composed of an apical and basal chamber, separated by a Polyethylene terephthalate (PET) membrane (pore size: $0.45 \mu \mathrm{m} ; 2 \times 10^{6}$ pores / cm²; ipCELLCULTURE, it4ip, Belgium). Both chambers are closed by two circular glass slides, one at the top and one at the bottom of the system, to allow live imaging. These are connected through fluidic tubing to separate medium reservoirs (the mixing chambers). The flow was generated by a peristaltic pump (LiveFlow, IVTech Srl, Italy).

Caco-2 cells (between passages 30 and 45) were seeded at a density of 25000 cells $/ \mathrm{cm}^{2}$ in bioreactors (through their tubing system) and PET 12-well-plate Transwell (pore size: $0.40 \mu \mathrm{m} ; 4$ x 106 pores / cm²; Corning, Italy). After cell adhesion, the apical chambers of the bioreactor were filled with $2 \mathrm{~mL}$ of medium and the basolateral ones with $1 \mathrm{~mL}$ of medium. In the Transwells, the apical and basolateral compartments were filled with $500 \mu \mathrm{L}$ and $1 \mathrm{~mL}$ of medium, respectively. All the samples were incubated at $37^{\circ} \mathrm{C}$ in a humidified atmosphere of $5 \% \mathrm{CO}_{2}$ for three weeks. In the static samples the medium was changed every three days.

After one week, the basal and apical circuits were filled up to a total volume of $10 \mathrm{~mL}$ of media respectively and kept in dynamic conditions (under flow) at a flow rate of $150 \mu \mathrm{L} / \mathrm{min}$, which according to the manufacturer should provide an average shear stress of around $6 \times 10^{-4} \mathrm{~Pa}$ on the membrane. In this set-up, the medium was changed once a week: half the volume was removed and replaced by fresh medium.

\section{Curcuma extract and APD compound preparation}

The extract of Turmeric was taken from a common encapsulated supplement (Curcumina Santé, from Santé Naturels, Italy) containing $450 \mathrm{mg}$ of pure curcumin, titled at $95 \%$. It is regularly registered as a 
food supplement notified to the Italian Ministry of Health. A stock solution of curcumin $(45 \mathrm{mg} / \mathrm{mL})$ was diluted in DMSO and stored at $-20^{\circ} \mathrm{C}$.

APD (kindly provided by the medicinal chemistry lab of Rapposelli Simona, Dept.of Pharmacy, UNIPI, Italy) was dissolved in Hank's Balanced Salt Solution (HBSS) and the stock solution (10 mM) was stored at $-20^{\circ} \mathrm{C}$. This compound belongs to a new class of molecules that showed appreciable modulatory activity on P-gp and a high degree of selectivity (17-19). The authors studied P-gp inhibition activity by 3 combined biological assays among which there was the inhibition of P-gp mediated transport of vinblastine. This derivative was shown to compete with radiolabeled vinblastine (a well-known P-gp substrate) for the P-gp binding site, with an $\mathrm{IC}_{50}$ value of $0.19 \mathrm{mM}$. (17).

\section{Assessment of curcumin toxicity}

The toxicity of curcumin was measured through a preliminary assessment of the metabolic activity of the cells, before and after incubation with the compound at different concentrations.

Caco- 2 cells were seeded in 24 well flat-bottom plates (Corning, Italy) and cultured for 7 days. At the seventh day the medium was replaced with fresh medium containing different concentrations of curcumin $(11.25,22.5,45,90 \mu \mathrm{g} / \mathrm{mL})$, assuring the DMSO was present at a non-toxic concentration $(0.02 \%)$. The cells were incubated with the different solutions at $37^{\circ} \mathrm{C}$ for 48 hours and the control samples solution contained only $0.02 \%$ DMSO in the cell medium.

\section{Cell barrier monitoring and morphological analysis}

Monolayer formation and maintenance were verified every three days with an optical microscope (Olympus AX81, Olympus Italy) both in the transwell and in the bioreactors. Moreover, transepithelial electric resistance (TEER) measurements, which are related to tight junction formations and thus provide an indication of cellular layer tightness, were performed.

Usually, TEER is measured applying a low-frequency $(\mathrm{f}<5 \mathrm{kHz})$ current stimulus across the cellular barrier and recording the resulting voltage $(16,20)$. In this study, it was monitored at $40 \mathrm{~Hz}$ with an integrated cellular impedance-meter $(16,21)$, in the bioreactors, and at $12.5 \mathrm{~Hz}$ with an Epithelial VoltOhmMeter (EVOM, World Precision Instruments, Florida USA) provided with chopsticks electrodes, in the Transwells. As reported in Cacopardo et al (16), the measurements performed by the two instruments are equivalent.

The final TEER values were obtained by subtracting the blank resistance values, related to the membrane and the culture medium, and multiplying by the surface area of the respective membrane (i.e. $1.2 \mathrm{~cm}^{2}$ for the TW and $1.8 \mathrm{~cm}^{2}$ for the bioreactors). Impedance spectra were also acquired in the bioreactors within a frequency range of $40-10000 \mathrm{~Hz}$, providing a more precise indication regarding the formation of a tight monolayer. TEEI values were obtained by subtracting the blank impedance values and multiplying by the membrane area. 


\section{Assessment of P-gp Activity}

On the twentieth day of culture, all the samples were rinsed with PBS and then incubated with a curcumin solution $(50 \mu \mathrm{g} / \mathrm{mL})$ that was added to the apical compartments through the mixing chambers in the bioreactors $(10 \mathrm{~mL})$ and directly in the apical compartment in the Transwells $(0.5 \mathrm{~mL})$. Basolateral compartments were filled fresh medium: $10 \mathrm{~mL}$ for the bioreactors and $1 \mathrm{~mL}$ for the Transwells. The flow rate was set to $150 \mu \mathrm{L} / \mathrm{min}$ in both circuits. All samples were incubated at $37^{\circ} \mathrm{C}$ overnight.

After the treatment, the curcumin solution was removed, and the samples were washed with PBS. The activity of P-gp was then evaluated using a fluorescent compound Rhodamine-123 (Rh-123) (SigmaAldrich, Italy), which is a tracer dye used as a substrate in the functional studies of MDR phenotype cells and in particular of the P-glycoprotein (22) (23). HBSS (ThermoFisher Scientific) was added in the apical compartments and the Rh-123 solution (10 $1 \mathrm{M}$ in HBSS) in the basolateral ones. The same procedure was carried out for the Transwell samples. At the time-points of 0 and 120-minutes, $100 \mu \mathrm{L}$ samples were collected from the apical sites and their volumes were replaced with pre-warmed HBSS. After two hours the samples were also collected from the basolateral ones. The Rh-123 concentration was determined using a plate reader (PerkinElmer, USA) setting filter wavelengths to $485 \mathrm{~nm}$ (excitation) and $535 \mathrm{~nm}$ (emission).

Positive and negative control experiments were performed, repeating the protocol. In the positive one APD $(100 \mu \mathrm{M})$ was added to the apical sites of models instead of curcumin. The aim of this test was to verify the inhibition of P-gp activity by the third-generation drug, together with the lower passage of Rh-123 on the apical side of the cells.Finally, a negative control to measure the basal activity of P-gp in physiological conditions, without any cell preconditioning was performed. All the experiments were conducted in parallel in the two in vitro models (dynamic and static) for each of the three conditions, with $\mathrm{n}=6$ experiments per condition. The three different experiments are illustrated in Figure 3.Prior to the cell studies, the same transport experiments in the both Transwells and bioreactors were performed in the absence of cells. The residual amount of Rh-123 that remained bound to the material was considered and used to normalize the values obtained with the cells.

\section{Data analysis}

The analysis of the effect of curcumin or APD on P-gp activity is based on Rh-123 efflux from the basolateral side to the apical side of planar cell cultures. Mathematically, P-gp activity is expressed as a function of Rh-123 mass $(\mu \mathrm{g})$ on both apical and basal sides at $120 \mathrm{~min}\left(\mathrm{t} 1_{\text {apical }}\right.$ and $\mathrm{t} 1_{\text {basal }}$, respectively), as well as Rh-123 initially administered to the experimental set-up ( $\left(\mathrm{t}_{\text {basal }}\right)$. All these parameters were normalized with respect to the blanks (i.e., measurements without cells) and combined into the equation as follows:

See formula 1 in the supplementary files. 
To appreciate how much the treatment with the substances (curcumin vs APD) inhibited the activity of the protein, the following equation was used:

\section{See formula 2 in the supplementary files.}

Statistics based on two-way ANOVA analysis was performed using the Tukey's test for multiple comparisons and setting statistical significance at $p<0.05$, with GraphPad Prism (GraphPad Software, San Diego, CA, USA). Error bars in results section represent standard deviations $(n=6)$.

\section{Results}

\section{Assessment of toxicity of curcumin}

The cytotoxicity of curcumin on Caco-2 cells was assessed by incubating the cells with different concentrations of curcumin. The cells were grown in a 24-well-plate for 7 days and their metabolic activity was determined by performing the Alamar blue assay, before and after $48 \mathrm{~h}$ incubation with the different curcumin extracts. The results (Figure 4A) showed that the metabolic activity of the cells was not substantially affected by curcumin solutions at the concentration range $45-11.25 \mu \mathrm{M}$. Only for the highest concentration tested, $90 \mu \mathrm{M}$, did the cell metabolic activity decrease down to roughly $70 \%$. A 50 $\mu \mathrm{M}$ curcumin solution was within the non-toxic range, so it was used for the P-gp activity modulation experiments.

\section{Cell monitoring and morphological analysis}

After the seeding in the bioreactors and in Transwells, Caco-2 cells were regularly monitored over a 21 day differentiation period, using an optical microscope and by measuring the TEER and TEEI.

The cells progressively formed a compact monolayer, exhibiting features of differentiated cells after 1012 days, as shown by the images in Figure 5 .

The formation of a compact monolayer was also verified by the TEER and TEEl analysis. As shown in Figure 4B, TEER increased during the culture reaching a plateau at day $14\left(\sim 800 \Omega . \mathrm{cm}^{2}\right.$ in the Transwells and $\sim 1000 \Omega . \mathrm{cm}^{2}$ in the bioreactors), and was maintained until day 21. Moreover, TEEl trends (Figure 4C), measured in the bioreactors, suggest the presence of an integral cell layer at all the time points (16).

After the P-gp modulation experiment, the cells were fixed and the actin fibers and the tight junction protein occludin were stained. The monolayer was maintained intact during the transport assay and the cells preserved their tight junctions, as pointed out by the presence of occludin (Figure 6A and 6C).

\section{Assessment of the activity of P-gp}


We measured P-gp activity as a function of Rh-123 efflux from the basal side to the apical side of cells cultured in the Transwell and in the bioreactors. Additionally, we assessed the modulation of curcumin and APD (i.e., positive control) by their inhibition effect.

Statistical analysis showed that in the absence of inhibitors P-gp activity observed in the bioreactors is lower than that measured in static conditions (Figure 4D). In the presence of natural and synthetic inhibitors, P-gp activity is further reduced in the bioreactors with respect to the Transwells (Figure 4E). Moreover, in both the static and dynamic conditions the inhibition of P-gp activity by curcumin (average inhibition values of $98 \%$ in static conditions and $100 \%$ in dynamic conditions) is higher than that observed in the positive controls (average inhibition values of $83 \%$ in static conditions and $92 \%$ in dynamic conditions) demonstrating that the natural substance is more markedly affects the Rh-123 efflux than the selective P-gp modulator APD.

\section{Discussion}

In the preliminary cytotoxicity test, we observed a decrease of Caco-2 metabolic activity ( $<20 \%)$ at the maximum curcumin concentration tested $(90 \mu \mathrm{M})$. Therefore, a non-toxic concentration of $50 \mu \mathrm{M}$ curcumin was used for the P-gp modulation experiment. Similar concentrations have been employed in other reports in the literature $(10,24,25)$.

Caco-2 cells grown in static Transwells and in bioreactors formed tight and well differentiated monolayers. It is worth noticing that the expansion of cells in the monolayer did not occur on a single plane, but gave rise to domes, as shown in Figure 5B and 5C, resembling the natural arrangement of cells in the intestinal environment (26). Both in the Transwells and in the bioreactors, it was possible to observe the progressive formation of the monolayer, which, as illustrated in Figure 4B, came to confluence in about seven days (TEER values higher than $600 \Omega . \mathrm{cm}^{2}$ ) reaching a maximum at day 14 and 21 ( $800 \Omega . \mathrm{cm}^{2}$ in the Transwells and $\sim 1000 \Omega . \mathrm{cm}^{2}$ in the bioreactors). The integrity of the monolayer in the bioreactors was confirmed by the TEEI measurements (Figure 4C), which follow a typical RC circuit trend. Indeed, the cell monolayer can be represented by an equivalent electric circuit composed of the resistive paracellular pathway and cell capacity (transcellular pathway) in parallel $(27,28)$. At lower frequencies the capacitor becomes progressively more charged and current flow across it decreases, thus ions flow paracellulary (i.e. through the tight junctions). The higher the density and tightness of tight junctions, the greater the low frequency resistance. At higher frequency, the capacitor becomes progressively more conductive and, in the presence of a compact cell layer with well-established tight junctions, the current is able to flow across the cells (transcellular current). The higher TEER values in the bioreactors with respect to transwells suggest that the fluidic conditions improve the barrier's integrity and tightness, as reported in Cacopardo et al. 2019 (16).

The presence of tight junctions was also confirmed by occluding staining, after 21 days in culture and after the passage test. Occludin is a protein that protrudes on the outer face of the membrane and mediates cell to cell adhesion. It has also been linked with the regulation of intermembrane and 
paracellular diffusion of small molecules (29). Furthermore, as demonstrated in Figure 6, the P-gp activity assessment experiment did not interfere with monolayer integrity and tightness.

The effect of curcumin on P-gp was evaluated through the efflux of Rhodamine-123 from the basal side to the apical side of the cells. Since P-gp is a membrane glycoprotein, expressed especially on the apical side of cells, the presence of Rhodamine-123 on the apical side can be correlated with its activity (30)(4).

Our results indicated that curcumin strongly inhibited P-gp activity in the static model, which is in line with the previously described studies performed in the absence of flow $(10,11)$. Using bioreactors, media flow was applied to intestinal cells at the liquid-liquid interface in an attempt to better mimic the in vivo environment. Our studies show that the inhibition effect of curcumin is even more marked in dynamic conditions. Both results reflect reports in mouse and rat models where curcumin was shown to affect the pharmacokinetics of orally administered and perfused anticancer drugs, through the reduction of P-gp activity $(31,32)$.

Regarding the inhibition by the synthetic modulator, data collected to date on APD shows that it inhibits the activity of P-gp at even lower doses than that used in this study (100 $\mu \mathrm{M})$ (Colabufo et al. 2008). Interestingly, we observed that the inhibition of P-gp by APD was surpassed by the inhibitory effect of curcumin (Figure 4E) in both static and dynamic conditions. As curcumin may also interact with other ABC-transporters expressed in Caco-2 cells (such as MRP2 and BCRP/ABCG2 $(33,34)$ ) for which APD does not show any activity (Colabufo et al. 2008), further investigations should be performed to confirm the higher inhibitory action of curcumin, with respect to APD on P-gp activity.

Regardless of the different potency of the two compounds towards P-gp, the main novelty of this study lies in the analysis of the effects of fluid flow on the apical and basolateral sides of the cells on the modulation of P-gp protein activity. The results show that there are notable differences in the passage and pumping of Rh-123 between the cells cultured on Transwell membranes and the cells cultured in the bioreactors and in all cases P-gp's efflux related activity was lower in dynamic conditions (Figures 4D and $4 \mathrm{E})$. This highlights the advantage of using bioreactors for Caco-2 models, since the drug efflux mediated by the P-gp is often overestimated in the traditional monolayer model (35).

We propose that the P-gp's efflux differences between static and dynamic conditions could be due to mechanotransduction effects arising from shear stress, which are known to modulate P-gp activity (36). Other studies have assessed the effect of fluid flow on P-gp expression, but not on its modulation using natural compounds. Based on the apparent permeability values reported by Pusch et al., the basal-apical transport of rhodamine in a 2D static Caco-2 culture was higher than in a dynamic 3D tissue equivalent. The authors did not compare 2D static and 2D dynamic conditions (26).

On the other hand, Deng et al (37) reported that Caco-2 cells cultured in a hollow fiber bioreactor revealed higher expression of P-gp, which was attributed to the topographic features of the substrate. Reporting a similar result, Schweinlin et al (38) observed that the expression of P-gp and efflux of Rh-123 increased in 
a decellularized biological scaffold-based multicellular model of the intestinal barrier cultured in a perfusion bioreactor, comparing with the values obtained for Caco-2 monoculture from another study.

It should be taken into account that the previously mentioned studies reported different bioreactors, substrates and/or cell source, arrangement and composition, which could justify the discrepancies in the events observed regarding P-gp activity, since the inclusion of different biochemical and mechanical cues can have potential synergistic effects (39). In any case, we can conclude that the presence of fluid flow impacts the functioning of the P-gp and needs to be considered in the development of physiologically relevant models of the intestine.

It is also worth noticing that differing events regarding the modulation of the protein by the presence of fluid flow have been reported not only in intestinal models. For instance, a study by Garcia-Polite et al demonstrated that the protein activity had a peak when endothelial cells were subjected to a shear stress of $1 \mathrm{~Pa}$ and that it decreased for a shear stress of $4 \mathrm{~Pa}(40)$; while there is a contrasting hypothesis that P-gp is down regulated in arterioles of rat brain in response to a higher shear stress $(41,42)$. Nevertheless, the shear stress provided by the flow in the present study is below the mentioned ranges and suggests that these cells are highly sensitive to even low levels of mechanical stimuli. It should however be noted that the analysis of the effects of increased shear stress are very difficult to decouple from those due to increased nutrient supply (43).

Remarkably, although the P-gp activity of the cells in the bioreactors was intrinsically lower, the activity inhibition efficiency, by both the ADP and curcumin, was higher in the bioreactors. This suggests that the presence of a flow not only influences P-gp by itself but also affects the action of external modulators. These contrasting events could be due to the effect of flow on the binding between substrate (Rh-123) and the protein and/or on the inhibition mechanism by both the Arylmethyloxy-phenyl derivative and curcumin.

It should be noted that the pharmacokinetic properties of curcumin in humans are not completely elucidated. It is known that when ingested, the substance has low oral bioavailability in humans (44) but it has been employed in several clinical trials of anticancer substances, with results reviewed in a paper by Kunnumakkara et al (45). The proposed dynamic model constitutes a more faithful representation of the microenvironment of the human intestinal epithelium in vitro, enabling better translation between in vivo and in vitro studies. At this point, further research is necessary to identify the precise mechanism by which P-gp modulation by curcumin was different in the dynamic system. Specifically, future in vitro studies should aim at assessing the intracellular localization of the P-gp in both static and dynamic conditions and to simulate the pharmacodynamic events curcumin undergoes before reaching and after translocating through the intestinal barrier.

\section{Conclusions}

We show that the presence of a physiological microenvironment can affect how P-gp is modulated by exogenous substances in vitro. Our results indicate that the inhibition of the protein by the action of 
curcumin is higher using a dynamic system compared with traditional static models. The potential therapeutic application of natural substances is of interest both in drug development and nutraceuticals, and it is crucial to perform in vitro studies using conditions which replicate the in vivo environment as faithfully as possible. As such, the present study is the first evidence that the use of more sophisticated tools can bring new insights on the therapeutic effects of natural substances such as curcumin.

\section{Declarations}

\section{Ethics approval and consent to participate}

Not applicable

\section{Consent for publication}

Not applicable

\section{Availability of data and materials}

All data generated or analysed during this study are included in this published article.

\section{Competing interests}

The authors declare that they have no competing interests.

\section{Funding}

Not applicable

\section{Authors' contributions}

AA, SR, and JJ conceived the work. AA supervised the work. JC, VA and LC performed the experiments. JC, LC and DP performed data analysis. JC, VA, LC and AA wrote the manuscript, with contributions from DP and SR.

\section{Acknowledgements}

Not applicable

\section{Authors' information (optional)}


Not applicable

\section{Abbreviations}

ABCG2: ATP binding cassette sub-family $\mathrm{G}$ member 2

APD: Arylmethyloxy-phenyl derivative

ATCC: American Type Culture Collection

ATP: Adenosine triphosphate

BCRP: Breast cancer resistance protein

Caco-2: Colorectal Adenocarcinoma cell line

DMSO: Dimethyl sulfoxide

EVOM: Epithelial VoltOhmMeter

FBS: Fetal Bovine Serum

HBSS: Hank's Balanced Salt Solution

LB2: LiveBox2 bioreactor

MDR: Multidrug Resistance

MRP2: Multidrug resistance-associated protein 2

P-gp: P-glycoprotein

PBS: Phosphate-buffered saline

PET: Polyethylene terephthalate

Rh-123: Rhodamine 123

TEEI: Transepithelial Electrical Impedance

TEER: Transepithelial Electrical Resistance

TW: Transwell

\section{References}


1. Lage H. An overview of cancer multidrug resistance: A still unsolved problem. Cellular and Molecular Life Sciences. 2008.

2. Gillet JP, Gottesman MM. Mechanisms of Multidrug Resistance in Cancer. In: Methods in Molecular Biology 596. 2010.

3. Pan GY, Wang GJ, Liu XD, Fawcett JP, Xie YY. The involvement of P-glycoprotein in berberine absorption. Pharmacol Toxicol. 2002;

4. Ho GT, Moodie FM, Satsangi J. Multidrug resistance 1 gene (P-glycoprotein 170): An important determinant in gastrointestinal disease? Gut. 2003.

5. Thomas $\mathrm{H}$, Coley HM. Overcoming multidrug resistance in cancer: An update on the clinical strategy of inhibiting P-glycoprotein. Cancer Control. 2003.

6. Benet LZ, Cummins CL. The drug efflux-metabolism alliance: biochemical aspects. Adv Drug Deliv Rev [Internet]. 2001;50 Suppl 1:S3-11. Available from: https://doi.org/10.1016/s0169409x(01)00178-8

7. Syed SB, Arya H, Fu IH, Yeh TK, Periyasamy L, Hsieh HP, et al. Targeting P-glycoprotein: Investigation of piperine analogs for overcoming drug resistance in cancer. Sci Rep. 2017;

8. Hu T, To KKW, Wang L, Zhang L, Lu L, Shen J, et al. Reversal of P-glycoprotein (P-gp) mediated multidrug resistance in colon cancer cells by cryptotanshinone and dihydrotanshinone of Salvia miltiorrhiza. Phytomedicine. 2014;

9. Nabekura T, Kamiyama S, Kitagawa S. Effects of dietary chemopreventive phytochemicals on Pglycoprotein function. Biochem Biophys Res Commun. 2005;

10. Ampasavate C, Sotanaphun U, Phattanawasin P, Piyapolrungroj N. Effects of Curcuma spp. on Pglycoprotein function. Phytomedicine. 2010;

11. Hou XL, Takahashi K, Tanaka K, Tougou K, Qiu F, Komatsu K, et al. Curcuma drugs and curcumin regulate the expression and function of P-gp in Caco-2 cells in completely opposite ways. Int $\mathrm{J}$ Pharm. 2008;

12. Hidalgo IJ, Raub TJ, Borchardt RT. Characterization of the human colon carcinoma cell line (Caco-2) as a model system for intestinal epithelial permeability. Gastroenterology [Internet]. 1989;96(3):73649. Available from: http://www.sciencedirect.com/science/article/pii/0016508589908974

13. Hilgers A, Conradi R, Burton P. Caco-2 cell monolayers as a model for drug transport across the intestinal mucosa. Pharm Res [Internet]. 1990;7(9):902-10. Available from:

http://dx.doi.org/10.1023/A:1015937605100

14. Larregieu CA, Benet LZ. Drug Discovery and Regulatory Considerations for Improving In Silico and In Vitro Predictions that Use Caco-2 as a Surrogate for Human Intestinal Permeability Measurements. AAPS J [Internet]. 2013;15(2):483-97. Available from: https://doi.org/10.1208/s12248-013-9456-8

15. Giusti S, Sbrana T, La Marca M, Di Patria V, Martinucci V, Tirella A, et al. A novel dual-flow bioreactor simulates increased fluorescein permeability in epithelial tissue barriers. Biotechnol J. 2014 Sep;9(9):1175-84. 
16. Cacopardo L, Costa J, Giusti S, Buoncompagni L, Meucci S, Corti A, et al. Real-time cellular impedance monitoring and imaging of biological barriers in a dual-flow membrane bioreactor. Biosens Bioelectron. 2019;140(March):111340.

17. Colabufo NA, Berardi F, Perrone R, Rapposelli S, Digiacomo M, Vanni M, et al. Synthesis and biological evaluation of (hetero)arylmethyloxy-and arylmethylamine-phenyl derivatives as potent Pglycoprotein modulating agents. J Med Chem. 2008;

18. Colabufo NA, Berardi F, Perrone R, Rapposelli S, Digiacomo M, Balsamo A. Arylmethyloxyphenyl Derivatives: Small Molecules Displaying P-Glycoprotein Inhibition. J Med Chem [Internet]. 2006 Nov 1;49(22):6607-13. Available from: https://doi.org/10.1021/jm060639z

19. Nesi G, Colabufo NA, Contino M, Perrone MG, Digiacomo M, Perrone R, et al. SAR study on arylmethyloxyphenyl scaffold: looking for a P-gp nanomolar affinity. Eur J Med Chem. 2014 Apr;76:558-66.

20. Srinivasan B, Kolli AR, Esch MB, Abaci HE, Shuler ML, Hickman JJ. TEER Measurement Techniques for In Vitro Barrier Model Systems. J Lab Autom. 2015;20(2):107-26.

21. Giusti S, Mazzei D, Cacopardo L, Mattei G, Domenici C, Ahluwalia A. Environmental control in flow bioreactors. Processes. 2017;5(2).

22. Twentyman PR, Rhodes T, Rayner S. A comparison of rhodamine 123 accumulation and efflux in cells with P-glycoprotein-mediated and MRP-associated multidrug resistance phenotypes. Eur $\mathrm{J}$ Cancer. 1994;

23. Tang F, Ouyang H, Yang JZ, Borchardt RT. Bidirectional Transport of Rhodamine 123 and Hoechst 33342, Fluorescence Probes of the Binding Sites on P-glycoprotein, across MDCK-MDR1 Cell Monolayers. J Pharm Sci. 2004;

24. Yue GGL, Cheng S-W, Yu H, Xu Z-S, Lee JKM, Hon P-M, et al. The Role of Turmerones on Curcumin Transportation and P-Glycoprotein Activities in Intestinal Caco-2 Cells. J Med Food. 2012;

25. Lu W, Qin Y, Yang C, Li L. Effect of curcumin on human colon cancer multidrug resistance in vitro and in vivo. Clinics. 2013;

26. Pusch J, Votteler M, Gohler S, Engl J, Hampel M, Walles H, et al. The physiological performance of a three-dimensional model that mimics the microenvironment of the small intestine. Biomaterials. 2011 Oct;32(30):7469-78.

27. Wegener J, Abrams D, Willenbrink W, Galla H-J, Janshoff A. Automated multi-well device to measure transepithelial electrical resistances under physiological conditions. Biotechniques. 2004 Oct;37(4):590,592-594,596-597.

28. Yeste J, Illa X, Alvarez M, Villa R. Engineering and monitoring cellular barrier models. J Biol Eng [Internet]. 2018;12(1):18. Available from: https://doi.org/10.1186/s13036-018-0108-5

29. Balda MS, Whitney JA, Flores C, González S, Cereijido M, Matter K. Functional dissociation of paracellular permeability and transepithelial electrical resistance and disruption of the apicalbasolateral intramembrane diffusion barrier by expression of a mutant tight junction membrane protein. J Cell Biol. 1996; 
30. Annese V, Valvano MR, Palmieri O, Latiano A, Bossa F, Andriulli A. Multidrug resistance 1 gene in inflammatory bowel disease: A meta-analysis. World Journal of Gastroenterology. 2006.

31. Lee C-K, Ki S-H, Choi J-S. Effects of oral curcumin on the pharmacokinetics of intravenous and oral etoposide in rats: possible role of intestinal CYP3A and P-gp inhibition by curcumin. Biopharm Drug Dispos [Internet]. 2011 May 1;32(4):245-51. Available from: https://doi.org/10.1002/bdd.754

32. Neerati P, Sudhakar YA, Kanwar JR. Curcumin Regulates Colon Cancer by Inhibiting P-Glycoprotein in In-situ Cancerous Colon Perfusion Rat Model. J Cancer Sci Ther. 2013 Jul;5:313-9.

33. Colabufo NA, Berardi F, Cantore M, Perrone MG, Contino M, Inglese C, et al. Small P-gp modulating molecules: SAR studies on tetrahydroisoquinoline derivatives. Bioorg Med Chem [Internet]. 2008;16(1):362-73. Available from: http://www.sciencedirect.com/science/article/pii/S0968089607008255

34. Maubon N, Le Vee M, Fossati L, Audry M, Le Ferrec E, Bolze S, et al. Analysis of drug transporter expression in human intestinal Caco-2 cells by real-time PCR. Fundam Clin Pharmacol. 2007 Dec;21(6):659-63.

35. Béduneau A, Tempesta C, Fimbel S, Pellequer Y, Jannin V, Demarne F, et al. A tunable Caco-2/HT29MTX co-culture model mimicking variable permeabilities of the human intestine obtained by an original seeding procedure. Eur J Pharm Biopharm [Internet]. 2014 Jul [cited 2014 Aug 21];87(2):290-8. Available from: http://www.ncbi.nlm.nih.gov/pubmed/24704198

36. Orr AW, Helmke BP, Blackman BR, Schwartz MA. Mechanisms of Mechanotransduction. Dev Cell [Internet]. 2006;10(1):11-20. Available from: http://www.sciencedirect.com/science/article/pii/S153458070500482X

37. Deng X, Zhang G, Shen C, Yin J, Meng Q. Hollow fiber culture accelerates differentiation of Caco-2 cells. Appl Microbiol Biotechnol. 2013 Aug;97(15):6943-55.

38. Schweinlin M, Wilhelm S, Schwedhelm I, Hansmann J, Rietscher R, Jurowich C, et al. Development of an Advanced Primary Human In Vitro Model of the Small Intestine. Tissue Eng Part C Methods. 2016 Sep;22(9):873-83.

39. Liang M-S, Koobatian M, Lei P, Swartz DD, Andreadis ST. Differential and synergistic effects of mechanical stimulation and growth factor presentation on vascular wall function. Biomaterials [Internet]. 2013/06/27. 2013 Oct;34(30):7281-91. Available from:

https://pubmed.ncbi.nlm.nih.gov/23810080

40. Garcia-Polite F, Martorell J, Del Rey-Puech P, Melgar-Lesmes P, O'Brien CC, Roquer J, et al. Pulsatility and high shear stress deteriorate barrier phenotype in brain microvascular endothelium. J Cereb Blood Flow Metab [Internet]. 2016/01/01. 2017 Jul;37(7):2614-25. Available from: https://www.ncbi.nlm.nih.gov/pubmed/27702879

41. Cho H, Lee H-Y, Han M, Choi J-R, Ahn S, Lee T, et al. Localized Down-regulation of P-glycoprotein by Focused Ultrasound and Microbubbles induced Blood-Brain Barrier Disruption in Rat Brain. Sci Rep [Internet]. 2016 Aug 11;6:31201. Available from: https://www.ncbi.nlm.nih.gov/pubmed/27510760 
42. Saubaméa B, Cochois-Guégan V, Cisternino S, Scherrmann J-M. Heterogeneity in the rat brain vasculature revealed by quantitative confocal analysis of endothelial barrier antigen and $\mathrm{P}$ glycoprotein expression. J Cereb Blood Flow Metab [Internet]. 2011/07/27. 2012 Jan;32(1):81-92. Available from: https://www.ncbi.nlm.nih.gov/pubmed/21792241

43. Mattei G, Giusti S, Ahluwalia A. Design Criteria for Generating Physiologically Relevant In Vitro Models in Bioreactors. Processes. 2014 Jul;2(3):548-69.

44. Sharma RA, McLelland HR, Hill KA, Ireson CR, Euden SA, Manson MM, et al. Pharmacodynamic and Pharmacokinetic Study of Oral Curcuma Extract in Patients with Colorectal Cancer. Clin Cancer Res [Internet]. 2001 Jul 1;7(7):1894 LP - 1900. Available from: http://clincancerres.aacrjournals.org/content/7/7/1894.abstract

45. Kunnumakkara AB, Bordoloi D, Harsha C, Banik K, Gupta SC, Aggarwal BB. Curcumin mediates anticancer effects by modulating multiple cell signaling pathways. Clin Sci (Lond). 2017 Aug;131(15):1781-99.

\section{Figures}
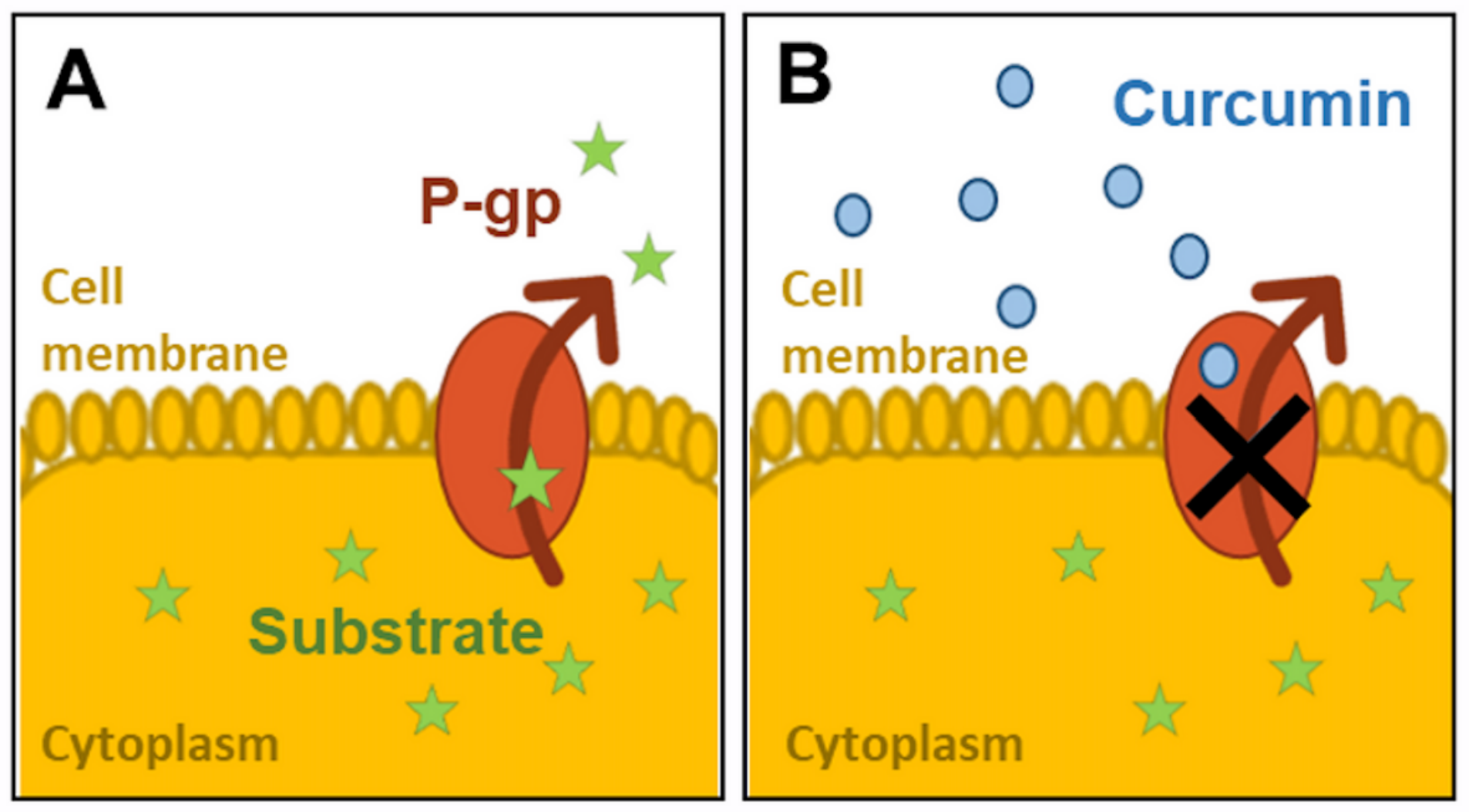

\section{Figure 1}

Inhibition of P-gp activity by Curcumin: A) The substrate is pumped out of the cell cytoplasm by P-gp; B) the activity of P-gp is inhibited by the presence of curcumin molecules on the apical surface. 


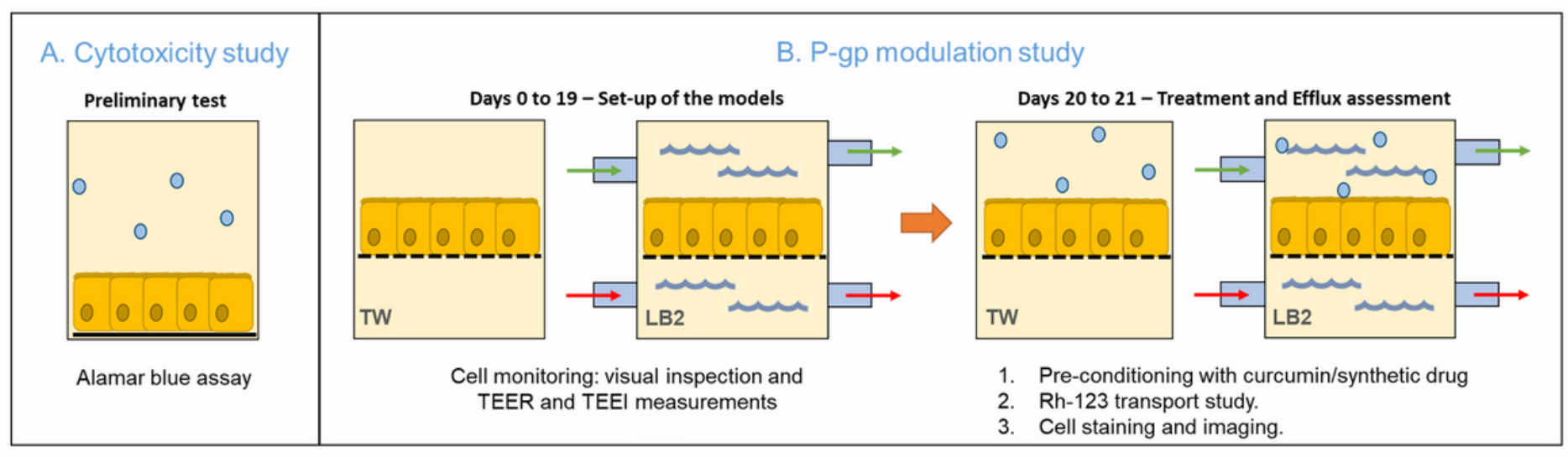

TW Transwell

LB2 Live-Box 2 bioreactor

O Compound

$\longrightarrow$ Apical flow $\longrightarrow$ Basal flow

\section{Figure 2}

Representation of the experimental approach. A) Preliminary study on the cytotoxicity of curcumin. B) Set up of the static and dynamic in vitro models and P-gp activity assessment at the end of the 21 days culture period.

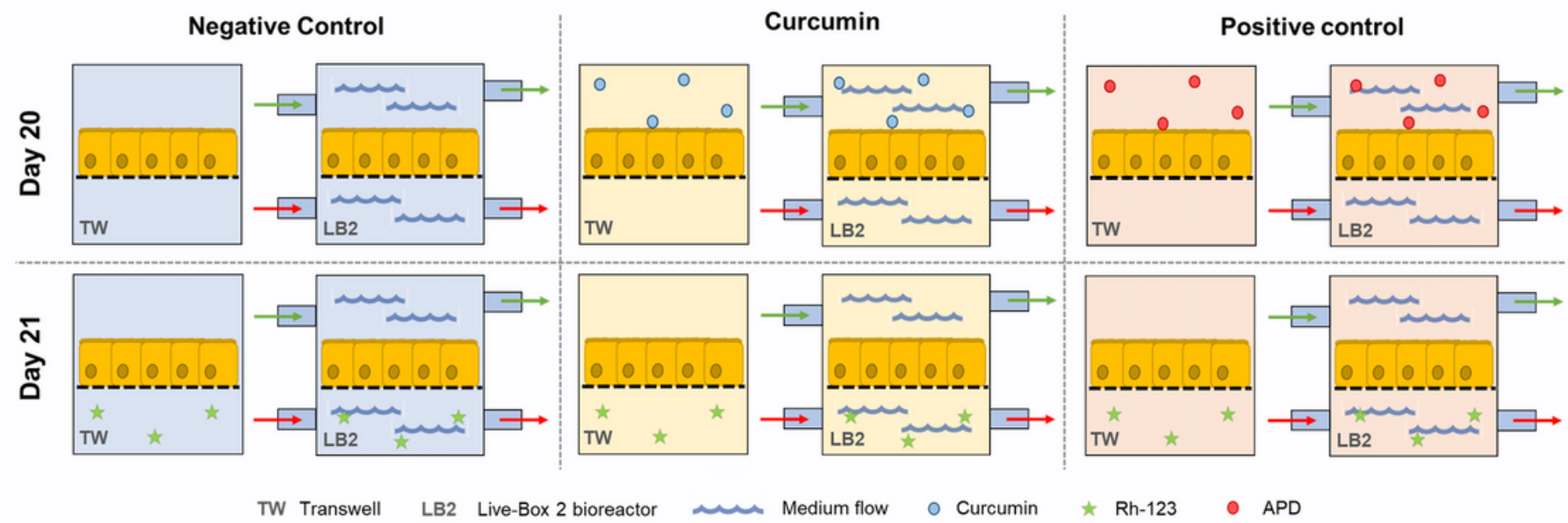

\section{Figure 3}

Schematic representation of the six different sample groups for the study of P-gp activity. 

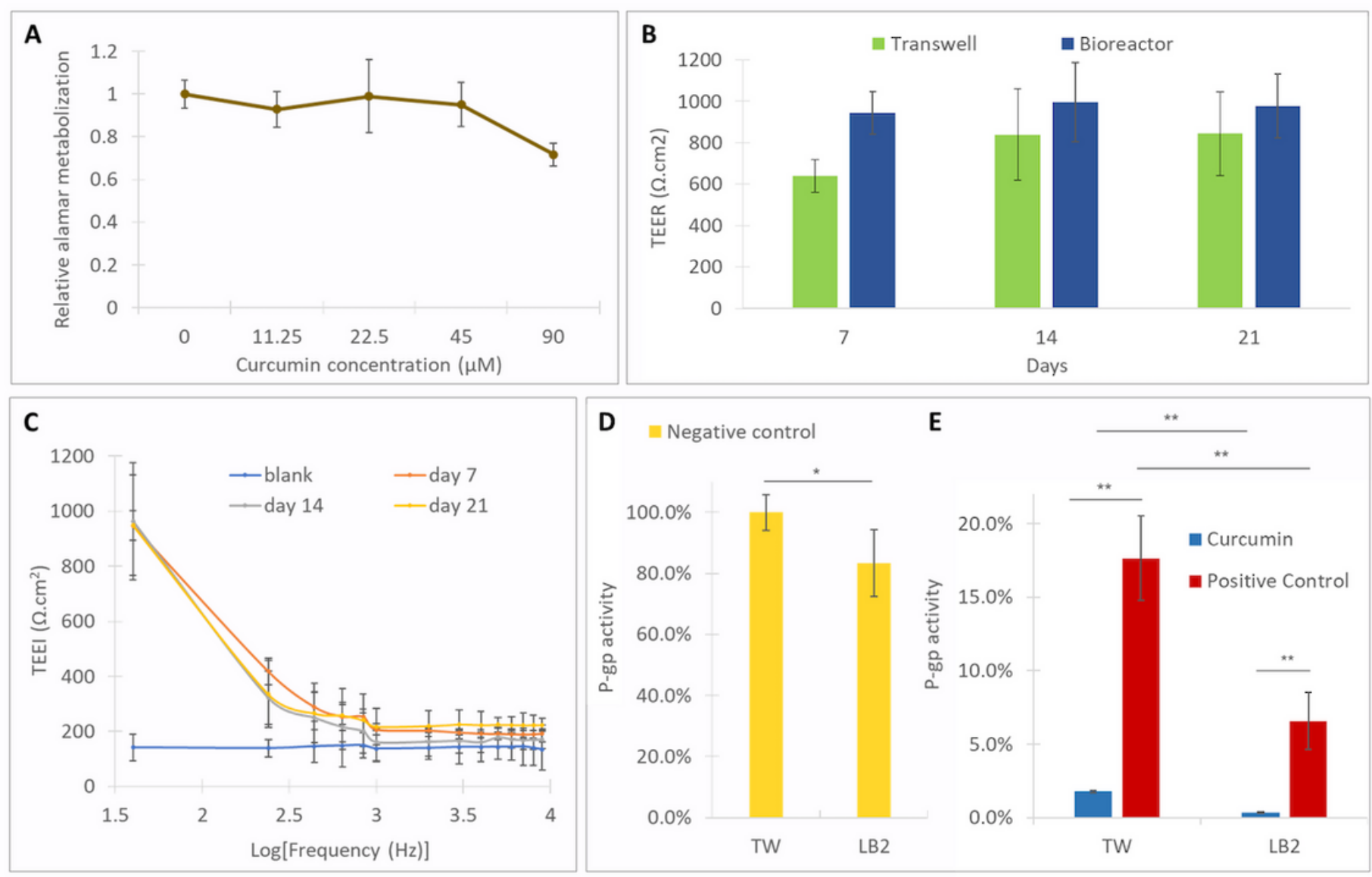

\section{Figure 4}

(A) Cell vitality with respect to $0 \mu \mathrm{M}$ control for different concentrations of curcumin after $48 \mathrm{~h}$ of incubation; Barrier monitoring through (B) TEER in the bioreactors and Transwells, (C) TEEl in the bioreactors at different timepoints; (D) P-gp activity in untreated samples (negative controls); (E) P-gp activity modulation by curcumin and APD (positive control) in Transwells and in the bioreactors. Error bars represent standard deviations $(n=6)$.
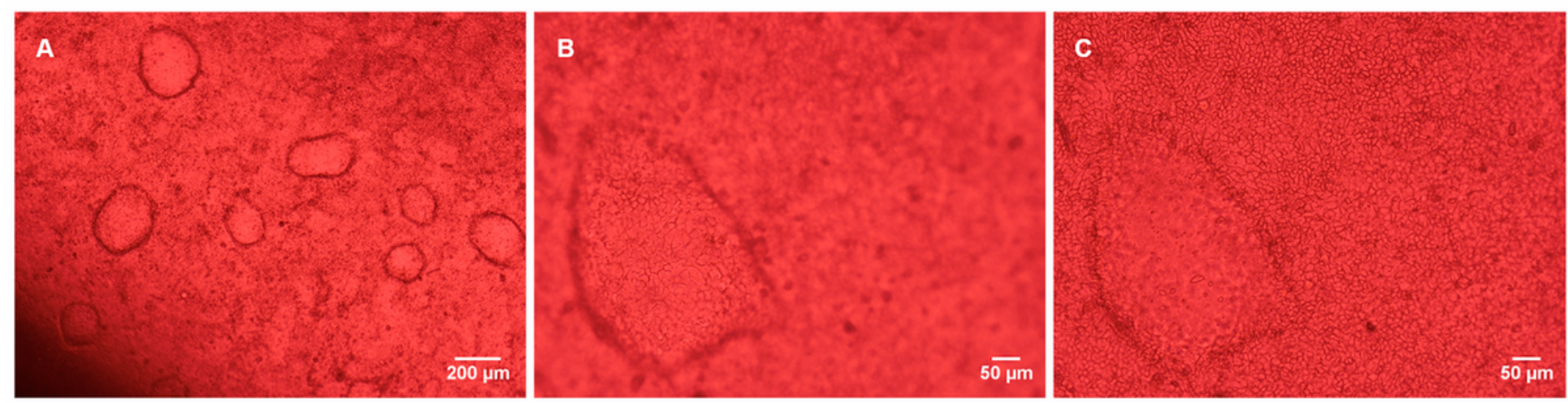

\section{Figure 5}


Caco-2 monolayer in the bioreactor at culture day 11. (A) 4x magnification; (B, C) 10x magnification (at different focal planes).
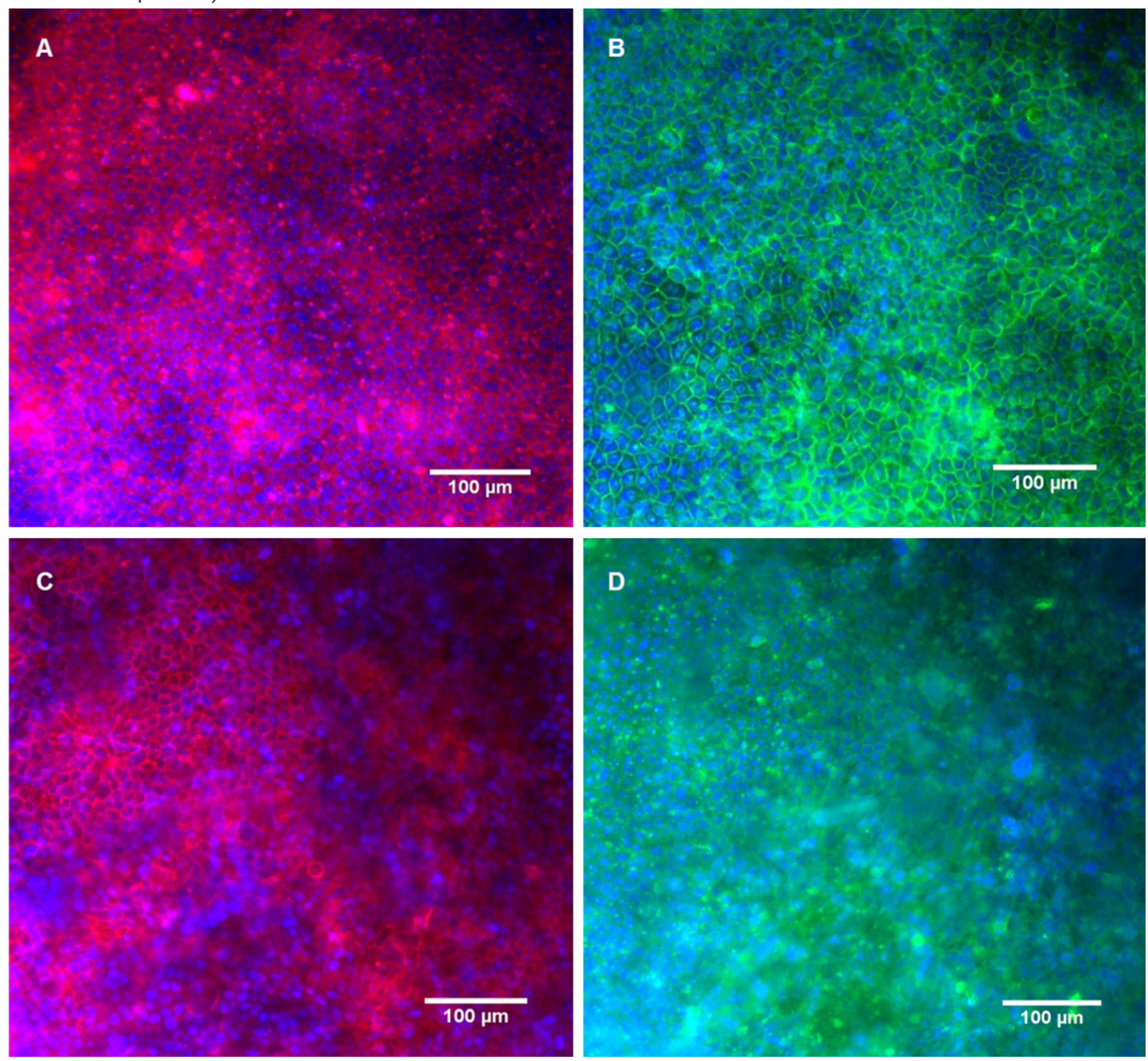

\section{Figure 6}

Caco-2 monolayer cultured in the bioreactor ( $A$ and $B$ ) and in the Transwells ( $C$ and $D$ ) after the P-gp activity assay. Fluorescence staining of the nuclei, actin microfilaments ( $A$ and $C$ ) and occludin (B and $D)$.

\section{Supplementary Files}

This is a list of supplementary files associated with this preprint. Click to download.

- formulas.docx 